\title{
Review
}

\section{Plant Lysophosphatidic Acids: A Rich Source for Bioactive Lysophosphatidic Acids and Their Pharmacological Applications}

\author{
Byung-Hwan Lee, ${ }^{a}$ Sun-Hye Choi, ${ }^{a}$ Hyeon-Joong Kim, ${ }^{a}$ \\ Seok-Won Jung, ${ }^{a}$ Ho-Kyoung Kim, ${ }^{b}$ and Seung-Yeol Nah*, \\ ${ }^{a}$ Ginsentology Research Laboratory and Department of Physiology, \\ College of Veterinary Medicine and Bio/Molecular Informatics Center, Konkuk University; \\ Seoul 143-701, Korea: and ${ }^{b}$ Mibyeong Research Center Korea Institute of \\ Oriental Medicine; Yuseong-gu, Daejeon 305-811, Republic of Korea. \\ Received July 23, 2015; accepted November 12, 2015
}

\begin{abstract}
Lysophosphatidic acid (1-acyl-2-lyso-sn-glycero-3-phosphatidic acid; LPA) is a simple and minor phospholipid in plants. Plant LPAs are merely metabolic intermediates in de novo lipid synthesis in plant cell membranes or for glycerophospholipid storage. The production and metabolisms of LPAs in animals are also well characterized and LPAs have diverse cellular effects in animal systems; i.e., from brain development to wound healing through the activation of $G$ protein-coupled LPA receptors. Recent studies show that various foodstuffs such as soybean, cabbage and seeds such as sesame and sunflower contain bioactive LPAs. Some LPAs are produced from phosphatidic acid during the digestion of foodstuff. In addition, herbal medicines such as corydalis tuber, and especially ginseng, contain large amounts of LPAs compared to foodstuffs. Herbal LPAs bind to cell surface LPA receptors in animal cells and exert their biological effects. Herbal LPAs elicit $\left[\mathrm{Ca}^{2+}\right]_{i}$ transient and are coupled to various $\mathrm{Ca}^{2+}$-dependent ion channels and receptor regulations via the activation of LPA receptors. They also showed beneficial effects of in vitro wound healing, in vivo antigastric ulcer, anti-Alzheimer's disease, autotaxin inhibition and anti-metastasis activity. Thus, herbal LPAs can be useful agents for human health. Humans can utilize exogenous plant-derived LPAs for preventive or therapeutic purposes if plant-derived LPAs are developed as functional foods or natural medicine targeting LPA receptors. This brief review article introduces the known rich sources of herbal LPAs and herbal LPA binding protein, describes their biological effects, and further addresses possible clinical applications.
\end{abstract}

Key words lysophosphatidic acid (LPA); plant LPA; LPA; LPA receptor; natural medicine

\section{INTRODUCTION}

Phospholipids are the main components of the plasma membrane lipid bilayer in animal and plant cells. ${ }^{1)}$ They are synthesized from glycerol, with fatty acids and a phosphate group conjugated to the glycerol backbone. Lysophosphatidic acids (LPAs) are one of the simplest of all glycerophospholipids. LPAs consist of three structural motifs: the phosphate head group, the glycerol linker and the hydrophobic tail fatty acid (Fig. 1). LPAs usually lack a fatty acid acyl-chain at the

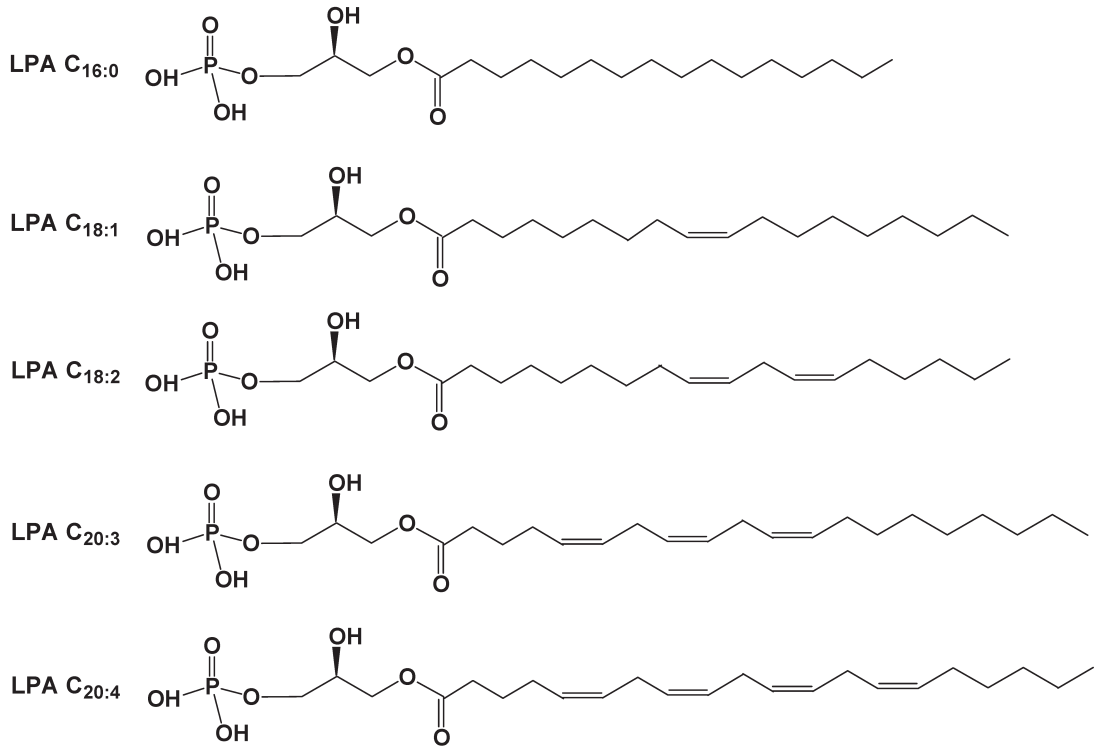

Fig. 1. Chemical Structure of Lysophosphatidic Acids (LPAs) Found in Animals and Plants 
2-position, containing only a hydroxyl group instead, which makes them designated "lysolipids" (Fig. 1). LPAs are also more water soluble because of their phosphate head and free hydroxyl group, another property that differs from other phospholipids. They are not lytic to cells, but a high concentration of LPAs in solution form micelles. ${ }^{2)}$ The phosphate head group of the glycerol backbone serves as a functional group for $G$ protein-coupled LPA receptor activation and hydrolysis of the phosphate head group causes a LPA receptor ligand functional loss. Unlike in LPAs, tail fatty acids are variable in diverse fatty acids. Long rather than short chain tail fatty acids are implicated in LPA receptor activation. ${ }^{3)}$ Thus, dependence on the number of carbon chains or on the degree of fatty acid saturation from saturation to polyunsaturation is also important for LPA receptor activation (Fig. 1).

Tokumura et l. $^{4)}$ first reported a novel component in a soybean lecithin fraction showing vasopressor activity in rats. ${ }^{4}$ In further studies, they determined that the unknown active ingredient in lecithin was LPA. LPA comprises about $0.1 \%$ of crude soybean lecithin and this was the first demonstration that plants also contain bioactive LPAs. In subsequent studies, they reported that lecithin-derived LPA exhibited almost the same activities as synthetic LPAs with regard to vasopressor activity, showing that LPAs derived from plants as well as animals are equally bioactive. ${ }^{5)}$ In plants, LPAs were identified in various parts such as leaf, seeds, and root but the content of LPAs in plants are variable. ${ }^{6-8)}$

Although it was unknown whether LPA receptor(s) are expressed in plant systems, Hecht et al. first reported that ventricular zone gene-1, which is abundantly expressed in the ventricular zone during mammalian brain neurogenesis, encodes the LPA1 receptor. ${ }^{9)}$ In subsequent studies, six LPA receptor subtypes were further cloned on the surface of various cells and most organs also expressed endogenous LPA receptor subtypes. ${ }^{1)}$ This is consistent with the diverse effects of LPA in animal systems including brain and vascular development, reproduction, and immune systems.

\section{SYNTHESIS OF LPAS IN PLANTS}

In plant cells, LPA is produced in intermediate processes during plant lipid biosynthesis. ${ }^{10)}$ De novo fatty acid biosynthesis first occurs in the plastid with the addition of two carbon units to a growing acyl-chain attached to a soluble acylcarrier-protein (ACP). Fatty acids are cleaved from the ACP by an acyl-ACP-thioesterase (TE) to form free fatty acids. These are exported to the cytoplasm, esterified to CoA, and join the acyl-CoA pool. The acyl groups are then used by the acyltransferases of the eukaryotic glycerol-3-phosphate pathway in the endoplasmic reticulum (ER) to produce membrane and storage lipids. The first acylation of glycerol-3-phosphate is catalyzed by glycerol-3-phosphate acyltransferase (GPAT), which acylates the $s n-1$ position of the glycerol backbone to produce plant lysophosphatidic acid (LPA) (Fig. 2). Subsequently, 1-acyl-glycerol-3-phosphate acyltransferase (LPAAT) acylates the $s n-2$ position to form phosphatidic acid (PA). Most LPA fatty acids are saturated $\left(\mathrm{C}_{16: 0}\right)$, monounsaturated $\left(\mathrm{C}_{18: 1}\right)$ or polyunsaturated $\left(\mathrm{C}_{18: 2}\right.$ or $\left.\mathrm{C}_{18: 3}\right)$ fatty acids linked to the glycerol backbone (Fig. 2, Table 1).

Thus, while the glycerol-3-phosphate pathway in the ER and the acyl-CoA pool in the cytoplasm are connected to LPA synthesis in the plant system, LPAs are produced through more diverse pathways in animal cells than in plant cells (Fig. 3, Table 1). This difference between animal and plant LPA synthesis might be due to additional evolutionary roles of LPA in the animal system beyond the role of a simple intermediate metabolite. LPAs are essential and critical for the development of the central nervous system ${ }^{2)}$ and are also involved in a variety of cellular effects in non-nervous systems in animals.

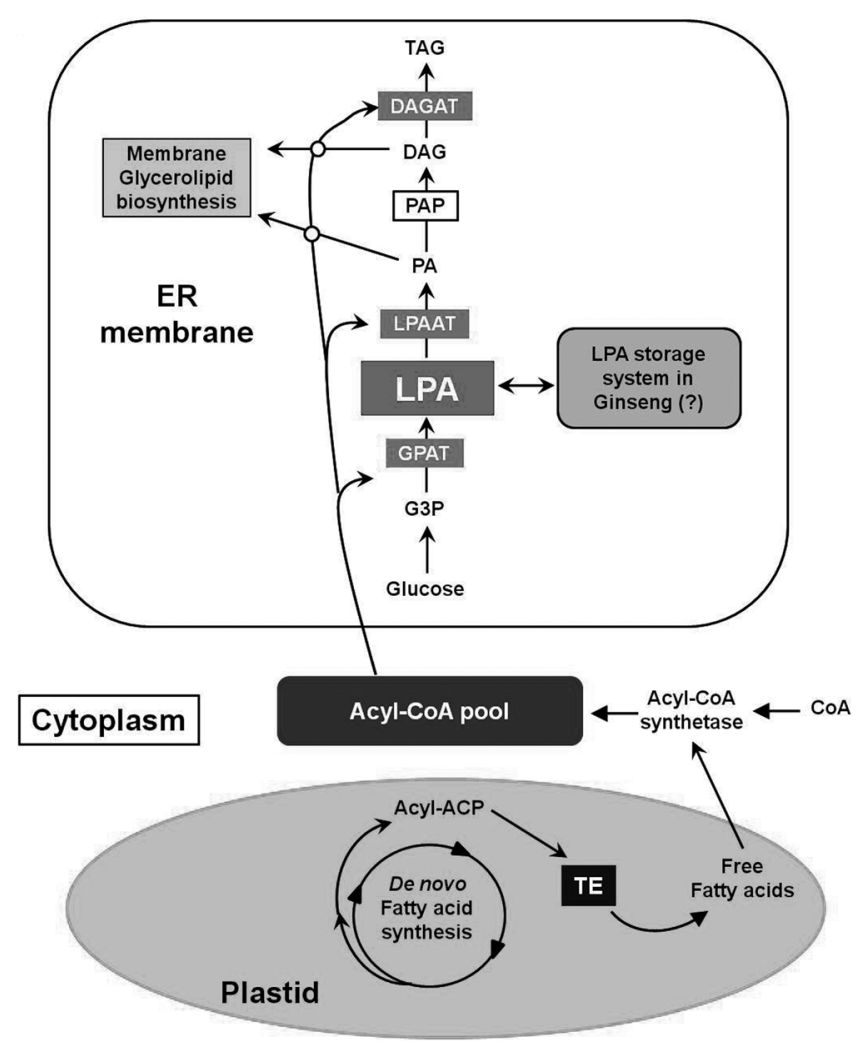

Fig. 2. A Simplified Diagram of LPA Synthesis in Plants

ACP, acyl-carrier-protein; DAGAT, diacylglycerol acyltransferase; G3P, glycerol3-phosphate; GPAT, glycerol-3-phosphate acyltransferase; LPA, lysophosphatidic acid; LPAAT, 1-acyl-glycerol-3-phosphate acyltransferase; PA, phosphatidic acid; PAP, phosphatidic acid phosphatase; TAG, triacylglycerol; TE, thioesterase. Since ginseng abundantly contains LPA compared to other plants, ginseng might have specific LPAs storage system and ginseng LPAs are present as a complex form with GLP151. This is needed to be proved in future study. This diagram was adapted from Millar et al. ${ }^{32)}$ with slight modifications.

Table 1. Comparison of LPA Precursors, Synthesis Sites, Enzymes, LPA Binding Proteins in Animal and Plant Cells

\begin{tabular}{|c|c|c|c|c|}
\hline & Precursors & Site of LPA synthesis & Enzymes & LPA binding proteins \\
\hline Animal cells & $\begin{array}{l}\text { Phosphatidic acid } \\
\text { glycerol-3-phosphate } \\
\text { lysophosphatidylcholine }\end{array}$ & $\begin{array}{l}\text { Plasma membrane } \\
\text { endoplasamic reticulum } \\
\text { or mitochondria plasma }\end{array}$ & $\begin{array}{l}\text { Secreted PLA2 } \\
\text { intracellular PLA1/2 } \\
\text { lysoPLD (autotaxin) }\end{array}$ & $\begin{array}{l}\text { Albumin, gelsolin and } \\
\text { intracellular fatty acid } \\
\text { binding protein }\end{array}$ \\
\hline Plant cells & Glycerol-3-phosphate & Endoplasmic reticulum & $\begin{array}{l}\text { Glycerol-3-phosphate } \\
\text { acyltransferase }\end{array}$ & GLP151 \\
\hline
\end{tabular}




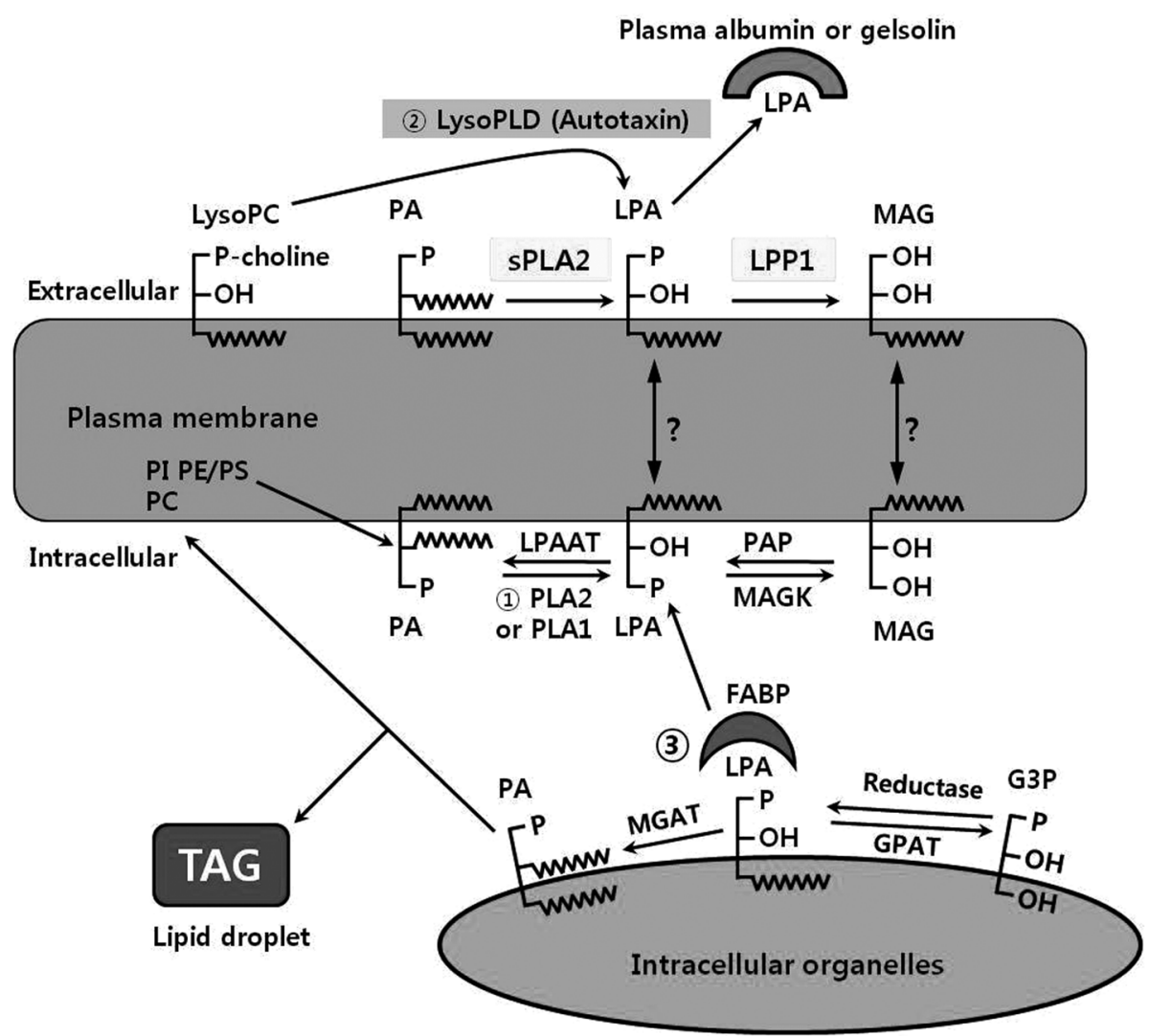

Fig. 3. A Simplified Diagram of LPA Synthesis in Animals

FABP, fatty acid binding protein; G3P, glycerol-3-phosphate; GPAT, glycerol-3-phosphate acyltransferase; LPA, lysophosphatidic acid; LPAAT, 1-acyl-glycerol-3-phosphate acyltransferase; LPP, lipid phosphate phosphatase; LysoPC, lysophosphatidylcholine; LysoPLD, lysophospholipase D; MAG, monoacylglycerol; MAGK, monoacylglycerol kinase; MGAT, monoacylglycerol acyltransferase; PA, phosphatidic acid; PAP, phosphatidic acid phosphatase; P-choline, phosphatidylcholine; PE, phosphatidylethanolamine; PI, phosphatidylinositol; PLA, phospholipase A; PS, phosphatidylserine; TG, triacylglycerol. This diagram was adapted from Pagès et al. ${ }^{33)}$ with slight modifications.

Table 1 summarizes LPA synthesis in plant cells and animal cells, with the LPA precursor, sites for LPA synthesis in cells and the enzymes involved.

\section{HERBAL MEDICINES AS RICH LPA SOURCES}

The soybean phospholipids-rich extract called lecithin contains previously unknown components that affect blood pressure in animals. These components have been identified as LPAs. It was also revealed that lecithin contains about $0.1 \%$ LPAs. ${ }^{5)}$ In addition, Tanaka et al. reported that vegetables such as cabbage, broccoli, komatsuna, green soybean, and radish contain LPAs and quantitated the LPA content using matrix-assisted laser desorption/ionization time-of-flight mass spectrometry (MALDI-TOF-MS). ${ }^{7}$ Among the vegetables, cabbage leaf contains the largest amount of LPAs followed by broccoli, komatsuna, and green soybean. Interestingly, the amount of LPA homologues in cabbage leaf was in the order of LPA $\mathrm{C}_{18: 3}>$ LPA $\mathrm{C}_{16: 0}>$ LPA $\mathrm{C}_{18: 2}>$ LPA $\mathrm{C}_{18: 1}$. The amount of LPA homologues in broccoli was in the order of LPA $\mathrm{C}_{18: 3}>$ LPA $\mathrm{C}_{16: 0}>$ LPA $\mathrm{C}_{18: 2}>$ LPA $\mathrm{C}_{18: 1}$ and the amount of LPA homologues in komatsuna was in the order of LPA $\mathrm{C}_{18: 3}>\mathrm{LPA}$ $\mathrm{C}_{16: 0}>$ LPA C $\mathrm{L}_{18: 2}>$ LPA C $18: 1$.

Extracts from seeds such as soybean, sunflower, and sesame also contain LPAs and these were quantitated using electrospray ionization (ESI)-MS/MS. Sesame contains a larger amount of LPAs than the other seeds examined. The amount of LPAs in sesame seeds is in the order of LPA $\mathrm{C}_{18: 2}>\mathrm{LPA}$
$\mathrm{C}_{16: 0}>$ LPA $\mathrm{C}_{18: 1}>$ LPA $\mathrm{C}_{18: 0}$. The amount of LPAs in sunflower seeds is in the order of LPA $\mathrm{C}_{18: 2}>$ LPA $\mathrm{C}_{16: 0}>$ LPA $\mathrm{C}_{18: 1}>\mathrm{LPA}$ $\mathrm{C}_{18: 0}$ while the amount of LPAs in soybean is in the order of LPA $\mathrm{C}_{18: 2}>$ LPA $_{16: 0}>$ LPA $\mathrm{C}_{18: 1}>$ LPA C $_{18: 0}{ }^{6}{ }^{6}$

Recently, there were two reports that herbal medicines containing corydalis tuber and ginseng also contain LPAs. A Chinese medicine called Antyu-san (AS) with anti-gastric ulcer activity consists of corydalis tuber, licorice, fennel, cinnamon bark, amomum seed, minor of galangal and oyster shell. Without corydalis tuber, AS did not show much anti-gastric ulcer activity and had much lower LPA content, indicating that LPAs are the main active ingredients behind AS-mediated anti-gastric ulcer activity. ${ }^{11)}$ The most abundant species in AS were LPA $C_{18: 2}>$ LPA $C_{16: 0}>$ LPA $C_{18: 1}>$ LPA $_{18: 3}>$ LPA $_{18: 0}$, determined through the analysis of MALDI-TOF-MS.

Ginseng is a traditional herbal medicine that is sometimes used as a general tonic. Although ginseng exhibits a variety of physiological and pharmacological activities, ginseng saponin (or ginsenoside) has no receptor of its own on the surface membrane of animal cells. Therefore, ginseng saponin alone cannot explain all of ginseng's actions and relatively little is known about the other active ingredients of ginseng. ${ }^{2)}$ Recent studies have shown that ginseng contains a unique form of LPAs, designated gintonin, which has been co-isolated with ginseng proteins such as ginseng major latex-like protein 151 and ginseng major storage protein. It seems that the complex of LPAs and ginseng proteins may provide some merit for the physiological and pharmacological actions of LPAs, since the 
Table 2. Comparisons of LPA Amounts among Plant Species

\begin{tabular}{|c|c|c|c|c|c|c|c|}
\hline Food stuffs & LPA C $_{16: 0}$ & LPA C ${ }_{18: 0}$ & LPA C $_{18: 1}$ & LPA C $18: 2$ & LPA $_{18: 3}$ & $\begin{array}{l}\text { Total LPAs } \\
\text { (nmol/g) }\end{array}$ & References \\
\hline Broccoli & $\approx 4.3$ & ND & $\approx 0.7$ & $\approx 4.0$ & $\approx 6.0$ & $\approx 15.0$ & 7) \\
\hline Cabbage leaves & $\approx 6.2$ & ND & $\approx 2$ & $\approx 5.8$ & $\approx 7.0$ & $21.0 \pm 6.0$ & 7) \\
\hline Komatsuna & $\approx 4.3$ & ND & $\approx 1.3$ & $\approx 3.6$ & $\approx 5.4$ & $\approx 14.6$ & 7) \\
\hline Sesame & $0.98 \pm 0.49$ & $<0.45$ & $0.46 \pm 0.23$ & $3.00 \pm 0.46$ & ND & 4.44 & 6) \\
\hline Soybean & $1.96 \pm 0.24$ & $<0.45$ & $0.92 \pm 0.23$ & $6.00 \pm 2.07$ & ND & 8.8 & 6) \\
\hline $\begin{array}{l}\text { Sunflower seed } \\
\text { Herbal medicines }\end{array}$ & $2.20 \pm 0.49$ & $<0.45$ & $1.84 \pm 0.46$ & $4.84 \pm 1.38$ & ND & 8.8 & 6) \\
\hline Corydalis tuber & 12.8 & ND & 5.1 & 14.7 & 1.9 & 34.5 & 11) \\
\hline Ginseng gintonin & $48.9 \pm 5.9$ & ND & BSL & $438 \pm 8.8$ & ND & 486 & 8) \\
\hline
\end{tabular}

BSL, below the sensitivity limit; ND, not determined.

Table 3. Biological Effects of Various Plant-Derived LPAs

\begin{tabular}{llc}
\hline \hline Plant LPAs & In vitro and in vivo biological effects & References \\
\hline Cabbage leaves & Wound healing & 7 ) \\
Corydalis tuber & Anti-gastric ulcer & $11)$ \\
Ginseng gintonin & Anti-Alzheimer's disease & $22)$ \\
& Anti-metastasis through inhibition of & $21)$ \\
& $\quad$ lysoPLD activity & \\
& Increase of gastrointestinal transit & $31)$ \\
& Increase of synaptic transmission & $25)$ \\
& Increase of gliotransmitter release & $26)$ \\
Sesame & Inhibition of lysoPLD activity & $6)$ \\
Soybean & Vasopressor or vasodepressor & $4,5)$ \\
& Inhibition of lysoPLD activity & $6)$ \\
Sunflower seed & Anti-gastric ulcer activity & $11)$ \\
\hline
\end{tabular}

free form of LPAs are labile to LPA hydrolysis enzymes such as lipid phosphate phosphatase. ${ }^{13)}$ Thus, the protein component might protect LPAs from hydrolysis in addition to storing and transporting LPAs to the protein's cognate receptors in target organs. The most abundant LPA species in gintonin were in the order of LPA $\mathrm{C}_{18: 2}>\mathrm{LPA} \mathrm{C}_{16: 0}>\mathrm{LPA} \mathrm{C}_{18: 1}$. Interestingly, ginseng contains an extraordinarily high amount of LPAs, which is 10 -fold greater than the amount in corydalis tuber and foodstuffs (Table 2).

The sum of the total amount of LPAs in herbal medicines, foodstuffs and hen egg is in order of ginseng gintonin $>$ AS $>$ cabbage $>$ broccoli $>$ komatsuna $>$ green soybean $>$ fertilized hen egg $>$ unfertilized hen egg. Interestingly, as one of the common features, the dominant species in foodstuffs- and traditional herbal medicines-derived LPAs is the polyunsaturated fatty acids in the tail of the LPA. The comparisons of LPA amounts among herbal medicines and plants are summarized in Table 2. Interestingly, most of LPA species found in plants are also commonly observed in animal systems. ${ }^{13)}$ Although LPA $\mathrm{C}_{18: 2}$ among LPAs isolated in some foodstuffs and herbal medicines was the most abundant, it might require more studies whether plant-derived LPA $\mathrm{C}_{18: 2}$ is closely correlated with biological effects in animal systems.

\section{LPA BINDING PROTEINS IN PLANT}

A recent study showed that ginseng major latex-like protein 151 (GLP151), a protein component of gintonin, also binds to LPA. ${ }^{14)}$ It was revealed through elucidation of the three dimen- sional structure of GLP151 that the phosphate head group of LPA binds to the imidazole ring of histidine residues at the C-terminal of GLP151 with hydrogen bonds and that the acylchain of LPA interacts with other portions of GLP151 since mutant GLP151 (mutation of histidine to alanine of GLP151) did not show affinity to LPA. ${ }^{14)}$ Thus, GLP151 is the first herbal medicine-derived protein that binds to LPA. This unique characteristic of gintonin LPAs might provide a distinguishing feature from the LPAs found in other foodstuffs and herbal medicine, since most of the LPAs derived from foodstuffs and herbal medicine are isolated in free form. This may be a great merit of ginseng gintonin as a stable LPA-rich source in herbal medicine.

\section{IN VITRO AND IN VIVO BIOLOGICAL ACTIVITIES OF LPAS IN FOODSTUFFS AND HERBAL MEDICINES}

Although it seems that plant LPAs in plants play a minor role as intermediate components of membrane composition or phospholipid storage (Fig. 2), herbal medicine-derived LPAs can exhibit diverse physiological and pharmacological effects in animal systems through the activation of $\mathrm{G}$ protein-coupled LPA receptors (Table 3). It was reported that unknown components of soybean lecithin regulate vasoactivity. ${ }^{4}$ Further studies revealed that the active ingredients in lecithin were LPAs. LPAs showed differential regulation of vasoactivity, which is dependent on animal species. For example, LPA shows vasopressor effects in cat and guinea pig, whereas LPA exhibited vasodepressor effects in cat and rabbit. ${ }^{5,15)}$ Thus, LPA actions on animal blood vessels might be dependent on the animal species, although the molecular mechanism underlying LPAs' induction of the opposite activity in the regulation of blood vessel activity remains unknown.

On the other hand, LPAs isolated from seeds such as sesame and sunflower and from soybean inhibit lysophospholipase D (LysoPLD) activity. ${ }^{6)}$ It is noteworthy that LPA $C_{18: 2}$ was the most potent LPA species in the inhibition of autotaxin (also called lysoPLD) activity. ${ }^{6,16)}$ It was reported that the ingestion of fresh cabbage reduced the healing period for ulcers of the stomach. ${ }^{17)}$ However, it was unknown which ingredients in cabbage were responsible for alleviating the effects of gastric ulcer. Tanaka et al. showed that LPAs from cabbage leaf induced the proliferation and migration of $3 \mathrm{~T} 3$ fibroblasts and also showed that this cell expresses endogenous LPA1, LPA2 and LPA4 receptor subtypes. ${ }^{7)}$ In addition, LPAs from cabbage increased the migration of the gastric epithelium-like cell line, 
HGC-27. Interestingly, chewing cabbage leaves in the mouth produces LPAs from cabbage phospholipids like phosphatidic acid and ingestion of cabbage leaves may help to heal gastric damages derived from various causes. Recently, Tanaka et al. showed that oral administration of LPA as well as phosphatidic acid attenuates aspirin-induced stomach mucosal injury, further supporting the idea that LPA could be used to attenuate aspirin side-effects in the stomach. ${ }^{18)}$ In addition, LPAs in soybean lecithin also exhibited anti-gastric ulcer activity. ${ }^{11)}$ In mechanism studies on anti-gastric ulceration of LPA, Tanaka et al. showed that LPA treatment enhanced prostaglandin E2 (PGE2) production stimulated by calcium ionophore A23187 in MKN74 cells, a gastric cell-line expressing LPA2 receptor. $^{16)}$ LPA increased the level of mRNA of cyclooxygenase (COX)-2 but not COX-1. However, LPA effects were abolished when the cells were pretreated with pertussis toxin (PTX), showing the involvement of receptor coupled with G $\alpha$ i protein. In addition, the inmmunofluorescent analysis using an antibody against LPA2 receptor showed that pit cells in gastric mucosa of mice express LPA2 receptor on the apical side of the plasma membrane. These results show that plant-derived LPAs may contribute to the epithelial integrity of stomach mucosa by enhancement of PGE2 production via activation of LPA2 receptor.

Herbal medicine-derived LPAs are relatively unknown components compared to other active ingredients with pharmacological activities. However, recent studies showed that LPAs in traditional herbal medicines play important roles in therapeutic actions. For example, AS is used to treat to gastric and duodenum ulcers, and gastritis. AS consists of licorice root, amomum seed, fennel, oyster shell, corydalis tuber, cinnamon bark, and lesser galagal. Among them, corydalis tuber is derived from Papareraceae plants that contain high phospholipase D activity and contains a large amount of LPA (Table 2), which is possibly produced from lysophospholipids by the phospholipase D activity. ${ }^{11,19)}$ AS prepared without corydalis tuber did not show anti-gastric ulcer activity. Thus, LPA components of corydalis tuber are main active ingredient in exhibiting anti-gastric ulcer activity. ${ }^{11)}$ These results indicated that foodstuffs-derived or herbal medicine-derived LPAs commonly exhibits anti-gastric ulcer activity through LPA receptor.

Regarding the anti-inflammatory activity of gintonin, gintonin attenuated the expression of proinflammatory cytokines in lipopolysaccharides (LPS)-stimulated RAW264.7 cells without cytotoxicity. Gintonin decreased nitric oxide (NO) production in LPS-stimulated RAW264.7 cells. Gintonin also decreased the mRNA expression of proinflammatory cytokines like IL-1 $\beta$, IL-6, iNOS, COX- 2 and TNF- $\alpha$. In addition, treatment of gintonin induced the same trend for protein expression of COX-2 and inducible NO synthase (iNOS) like NO production the Western blot analysis, confirming the anti-inflammatory effects of gintonin. Gintonin used the nuclear factor kappa$\mathrm{B}(\mathrm{NF}-\kappa \mathrm{B})$ and mitogen-activated protein kinases (MAPK) signaling pathways. Gintonin restored the levels of mir-34a and mir-93 that were reduced by LPS. Thus, gintonin exhibits anti-inflammatory effects via $\mathrm{NF}-\kappa \mathrm{B}$ and MAPK signaling pathways. $^{20)}$

It was revealed that ginseng also contains LPAs. LPAs in ginseng gintonin exhibit their biological effects in four ways: one is the inhibition of autotoxin activity and the other is through LPA receptor activation. First, gintonin strongly inhibits lysoPLD (autotaxin) activity in melanoma cells in addition to inhibiting cell motility and migration. However, gintonin has almost no effects on cell proliferation. In addition, oral administration of gintonin inhibited metastasis to the lungs after administration of the cells to the tail vein and inhibited tumor growth after transplantation of melanoma cells under the skin in mouse. ${ }^{21)}$ Second, gintonin showed anti-Alzheimer's disease activity through the activation of the non-amyloidogenic pathway via LPA receptors. Gintoninmediated LPA receptor activation is coupled to the increase of soluble amyloid precursor protein $\alpha(\operatorname{sAPP} \alpha)$ release instead of $\beta$-amyloid in neuroblastoma SH-SY5Y cells. ${ }^{22)}$ In addition, long-term oral administration of gintonin attenuated neuropathies of $\beta$-amyloid plaques in the cortex and hippocampus and restored $\beta$-amyloid-induced memory dysfunctions. Third, long-term administration of gintonin to wild-type mice increased the immunoreactivity of hippocampal choline acetyltransferase, which is responsible for increase of acetylcholine synthesis. ${ }^{23)}$ In behavioral tests, gintonin treatment restored scopolamine-induced memory dysfunction in passive avoidance and Morris water maze tests. ${ }^{23)}$ Thus, LPAs in gintonin contributed to the restoration of adult brain cholinergic dysfunctions following neurodegeneration. ${ }^{22}$ Fourth, Kim et al. observed that hippocampal long-term potentiation (LTP) increased in mice that were previously treated with gintonin for $7 \mathrm{~d}$ orally compared to saline-treated mice. ${ }^{24)}$ In addition, the hippocampi of mice that were previously treated for $7 \mathrm{~d}$ with gintonin showed increased expressions of learning and memory-related proteins such as phosphorylated cAMP-response element binding ( $\mathrm{pCREB}$ ) protein and brain-derived neurotrophic factor (BDNF). Finally, in a behavioral study, gintonin administration improved fear memory retention in the contextual fear-conditioning test in mice. ${ }^{23)}$ Therefore, long-term oral administration of gintonin decreases amyloid $\beta(\mathrm{A} \beta)$ formation and enhances cognitive functions via activations of the cognition related proteins and the cholinergic system.

Park et al. reported that gintonin enhanced both excitatory and inhibitory transmission in the central synapses of adult rat hippocampal slices via LPA receptors and showed that gintonin-mediated LPAR activation primarily resulted in synaptic enhancement by activating postsynaptic $N$-methyl-D-aspartate (NMDA) and $\alpha$-amino-3-hydroxy-5-methyl-4isoxazolepropionic acid (AMPA) receptors. ${ }^{25}$ ) These results showed that gintonin as an exogenous LPA is able to directly potentiate synaptic transmission in hippocampal synapses. In primary cortical astrocytes, gintonin induces a $\left[\mathrm{Ca}^{2+}\right]_{\mathrm{i}}$ transient via LPA receptor signaling pathways. Gintonin as well as LPA stimulates the release of gliotransmitters such as ATP and glutamate, and this effect is also $\left[\mathrm{Ca}^{2+}\right]_{i}$-sensitive because intracellular $\mathrm{Ca}^{2+}$ chelator abolishes gintonin-mediated gliotransmitter release. ${ }^{26)}$ Thus, exogenous ginseng-derived LPAs could regulate synaptic transmission in the adult hippocampus as well as gliotransmitter release. Finally, herbal medicinederived regulation of LPA receptors can serve to modulate synaptic activity and gliotransmitter release to exert beneficial effects in neurodegenerative diseases. In vitro and in vivo biological activities of herbal medicine and plant-derived LPAs are summarized in Table 3.

Ginseng has two distinguishing characteristics in that it has a large amount of LPAs and these LPAs are not found in the 


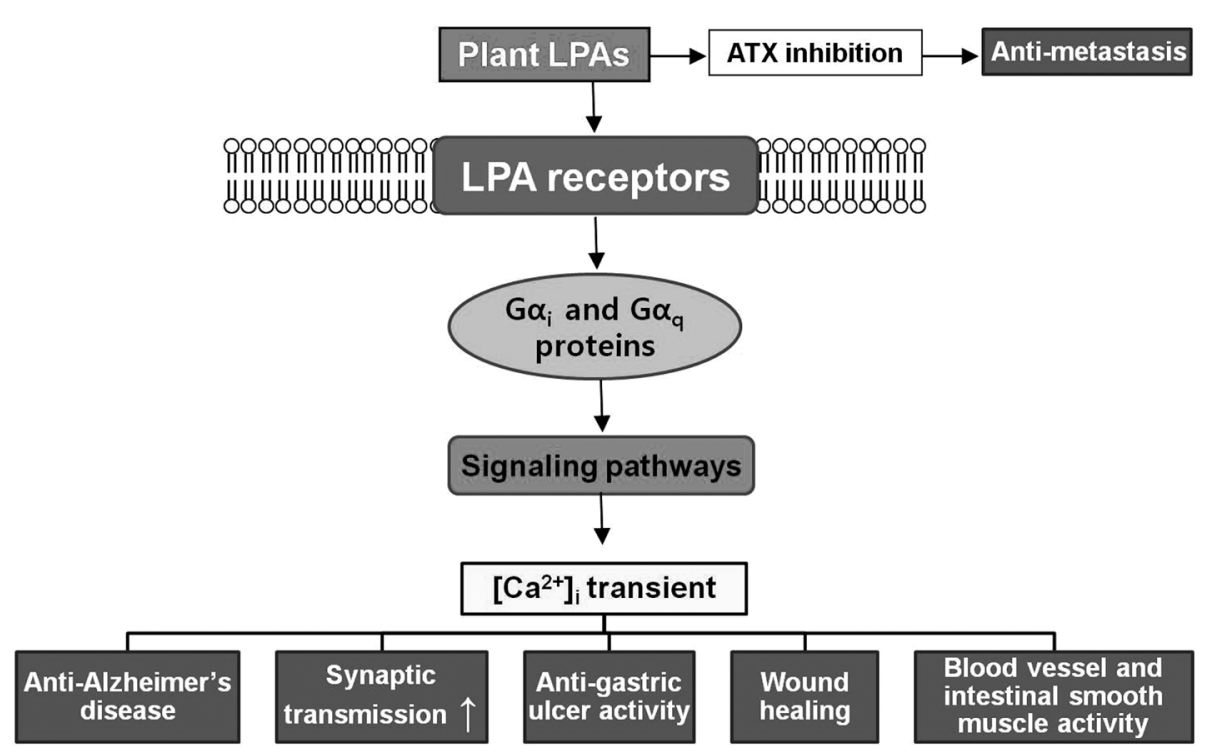

Fig. 4. The Signaling Pathways and Biological Effects of Herbal Medicine-Derived LPA in Animal Systems

free form. It is interesting to consider why ginseng contains a much larger amount of LPAs (especially LPA $\mathrm{C}_{18: 2}$ ) in the form of complexes of LPAs and ginseng proteins compared to other food stuffs and herbal medicines. One possible assumption is that ginseng might contain a differential system for LPA synthesis and storage, unlike other plants or herbal medicines. Ginseng may have two pathways for LPA synthesis and storage. One would be the usual pathway for the utilization of LPA as an intermediate metabolite for glycerophospholipid synthesis. The other system may involve a specific LPA storage pathway to form complexes with ginseng proteins. The other characteristic is that LPAs in ginseng are not in free form as noted above. The complexes of ginseng proteins and LPAs may become concentrated as the ginseng grows, similar to other lipid droplets, ${ }^{27)}$ although the presence, location of storage, and the roles of complexes need to be explained in the future. Figure 2 shows a hypothetical schematic drawing of ginseng LPA synthesis and storage in the form of gintonin.

\section{CONCLUDING REMARKS AND PERSPECTIVES}

Tokumura et al. first reported the presence of LPA in soybean lecithin. ${ }^{5)}$ Plant systems synthesize LPAs and herbal medicine-derived LPAs can also serve as G protein-coupled LPA receptor ligands in animal cells. However, tissue distribution, content, cells and subcellular compartments of LPAs are still unknown. In the future, applications of plant lipidomics for plant LPAs could provide additional information for LPA localization of plant cells. ${ }^{28)}$

In the beginning of LPA and LPA receptor-related studies, it seemed that LPA receptors were expressed in limited amounts in the mammalian developing brain. However, with the discovery of LPA receptor subtypes in various animal organs, most organs have been found to express LPA receptors including organs in the nervous system, cardiovascular system, immune system, and endocrine and reproductive system. ${ }^{1)}$ In each organ, LPA plays an important role for cell proliferation, migration, division and survival. However, the availability of endogenous LPA in animal cells or body fluids is very low because of its low concentration. If exogenous
LPAs derived from herbal medicines or plants could be applied to animal systems, it will facilitate or promote various LPA receptor-related biological effects. This review provides several representative examples that exogenous plant-derived LPAs are involved in anti-Alzheimer's disease, enhancement of synaptic transmission, regulations of smooth muscle activity, anti-gastric activity, wound healing, and anti-metastasis activity in animal systems via LPA receptor signal transduction pathway or regulations of autotaxin (ATX) activity (Fig. 4). However, it needs to pay an attention to use plant-derived LPAs in pathophysiological conditions such as cancers of breast cancer, melanoma, and ovarian cancer, since autotaxin activity is increased in these cancer patients. ${ }^{29)}$ These examples might not be enough for pharmacological or clinical applications of plant-derived LPAs however. In the future, more studies on LPA-derived biological effects could expand the applications of herbal LPAs. In addition, it is currently unknown how LPAs are absorbed in the gastrointestinal system, although orally administered herbal medicine-derived LPAs exhibit biological effects. ${ }^{17,21,22)}$ Therefore, further studies will be required to elucidate the pharmacokinetics of plant-derived exogenous LPA in animal systems.

In conclusion, food-stuffs and herbal medicines contain various beneficial ingredients for keeping humans healthy from diseases. LPAs could be one of these ingredients. Herbal medicine like ginseng may be major sources of LPAs. With chemical synthesis of LPA analogs for the development of LPA receptor agonist or antagonist, ${ }^{30)}$ it might be worthwhile to pay attention to herbal medicine-derived LPAs. In the future, herbal medicine-derived LPAs that target human LPA receptors could be developed as functional foods or as natural medicines for human health.

Acknowledgments This work was supported by the Basic Science Research Program (NRF-2014R1A1A2054538) which is funded by the Ministry of Education, Science, and Technology (2012-0006686) and by the BK21 plus project fund to S.-Y. Nah.

Conflict of Interest The authors declare no conflict of 
interest.

\section{REFERENCES}

1) Yung YC, Stoddard NC, Chun J. LPA receptor signaling: pharmacology, physiology, and pathophysiology. J. Lipid Res., 55, 11921214 (2014)

2) Li Z, Mintzer E, Bittman R. The critical micelle concentrations of lysophosphatidic acid and sphingosylphosphorylcholine. Chem. Phys. Lipids, 130, 197-201 (2004).

3) Tigyi G, Parrill AL. Molecular mechanisms of lysophosphatidic acid action. Prog. Lipid Res., 42, 498-526 (2003).

4) Tokumura A, Fukuzawa K, Akamatsu Y, Yamada S, Suzuki T, Tsukatani H. Identification of vasopressor phospholipid in crude soybean lecithin. Lipids, 13, 468-472 (1978).

5) Tokumura A, Fukuzawa K, Tsukatani H. Effects of synthetic and natural lysophosphatidic acids on the arterial blood pressure of different animal species. Lipids, 13, 572-574 (1978).

6) Liu XW, Sok DE, Yook HS, Sohn CB, Chung YJ, Kim MR. Inhibition of lysophospholipase D activity by unsaturated lysophosphatidic acids or seed extracts containing 1-linoleoyl and 1-oleoyl lysophosphatidic acid. J. Agric. Food Chem., 55, 8717-8722 (2007).

7) Tanaka T, Horiuchi G, Matsuoka M, Hirano K, Tokumura A, Koike T, Satouchi K. Formation of lysophosphatidic acid, a wound-healing lipid, during digestion of cabbage leaves. Biosci. Biotechnol. Biochem., 73, 1293-1300 (2009).

8) Hwang SH, Shin TJ, Choi SH, Cho HJ, Lee BH, Pyo MK, Lee JH, Kang J, Kim HJ, Park CW, Shin HC, Nah SY. Gintonin, newly identified compounds from ginseng, is novel lysophosphatidic acids-protein complexes and activates G protein-coupled lysophosphatidic acid receptors with high affinity. Mol. Cells, 33, 151-162 (2012).

9) Hecht JH, Weiner JA, Post SR, Chun J. Ventricular zone gene-1 (vzg-1) encodes a lysophosphatidic acid receptor expressed in neurogenic regions of the developing cerebral cortex. J. Cell Biol., 135, 1071-1083 (1996).

10) Millar AA, Smith MA, Kunst L. All fatty acids are not equal: discrimination in plant membrane lipids. Trends Plant Sci., 5, 95-101 (2000).

11) Adachi M, Horiuchi G, Ikematsu N, Tanaka T, Terao J, Satouchi K, Tokumura A. Intragastrically administered lysophosphatidic acids protect against gastric ulcer in rats under water-immersion restraint stress. Dig. Dis. Sci., 56, 2252-2261 (2011).

12) Nah SY, Kim DH, Rhim H. Ginsenosides: are any of them candidates for drugs acting on the central nervous system? CNS Drug Rev., 13, 381-404 (2007).

13) Salous AK, Panchatcharam M, Sunkara M, Mueller P, Dong A, Wang Y, Graf GA, Smyth SS, Morris AJ. Mechanism of rapid elimination of lysophosphatidic acid and related lipids from the circulation of mice. J. Lipid Res., 54, 2775-2784 (2013).

14) Choi SH, Hong MK, Kim HJ, Ryoo N, Rhim H, Nah SY, Kang LW. Structure of ginseng major latex-like protein 151 and its proposed lysophosphatidic acid-binding mechanism. Acta Crystallogr. D Biol. Crystallogr., 71, 1039-1050 (2015).

15) Schumacher KA, Classen HG, Späth M. Platelet aggregation evoked in vitro and in vivo by phosphatidic acids and lysoderivatives: identity with substances in aged serum (DAS). Thromb. Haemost., 42, 631-640 (1979).

16) Tanaka T, Ohmoto M, Morito K, Kondo H, Urikura M, Satouchi $\mathrm{K}$, Tokumura A. Type 2 lysophosphatidic acid receptor in gastric surface mucous cells: Possible implication of prostaglandin E2 production. Biofactors, 40, 355-361 (2014).
17) Cheney G. Rapid healing of peptic ulcers in patients receiving fresh cabbage juice. Calif. Med., 70, 10-15 (1949).

18) Tanaka T, Morito K, Kinoshita M, Ohmoto M, Urikura M, Satouchi K, Tokumura A. Orally administered phosphatidic acids and lysophosphatidic acids ameliorate aspirin-induced stomach mucosal injury in mice. Dig. Dis. Sci., 58, 950-958 (2013).

19) Oblozinsky M, Schoeps R, Ulbrich-Hofmann R, Bezakova L. Two uncommon phospholipase D isoenzymes from poppy seedlings (Papaver somniferum L.). Biochim. Biophys. Acta, 1631, 153-159 (2003).

20) Saba E, Jeon BR, Jeong DH, Lee K, Goo YK, Kwak D, Kim S, Roh SS, Kim SD, Nah SY, Rhee MH. Gintonin from Korean Red Ginseng suppresses inflammation in RAW264.7 cells by targeting $\mathrm{NF}-\kappa \mathrm{B}$ and revives the levels of mir-34a and mir-93. Evid. Based Complement. Alternat. Med., 2015, 624132 (2015).

21) Hwang SH, Lee BH, Kim HJ, Cho HJ, Shin HC, Im KS, Choi SH, Shin TJ, Lee SM, Nam SW, Kim HC, Rhim H, Nah SY. Suppression of metastasis of intravenously-inoculated B16/F10 melanoma cells by the novel ginseng-derived ingredient, gintonin: involvement of autotaxin inhibition. Int. J. Oncol., 42, 317-326 (2013).

22) Hwang SH, Shin EJ, Shin TJ, Lee BH, Choi SH, Kang J, Kim HJ, Kwon SH, Jang CG, Lee JH, Kim HC, Nah SY. Gintonin, a ginseng-derived lysophosphatidic acid receptor ligand, attenuates Alzheimer's disease-related neuropathies: involvement of nonamyloidogenic processing. J. Alzheimer's Dis., 31, 207-223 (2012).

23) Kim HJ, Shin EJ, Lee BH, Choi SH, Jung SW, Cho IH, Hwang SH, Kim JY, Han JS, Chung C, Jang CG, Rhim H, Kim HC, Nah SY. Oral administration of gintonin attenuates cholinergic impairments by scopolamine, amyloid- $\beta$ protein, and mouse model of Alzheimer's disease. Mol. Cells, 38, 796-805 (2015).

24) Kim S, Kim MS, Park K, Kim HJ, Jung SW, Nah SY, Han JS, Chung CH. Hippocampus-dependent cognitive enhancement induced by systemic gintonin administration. J. Ginseng Res. (2015), in press.

25) Park H, Kim S, Rhee J, Kim HJ, Han JS, Nah SY, Chung CH Synaptic enhancement induced by gintonin via lysophosphatidic acid receptor activation in central synapses. J. Neurophysiol., 113, 1493-1500 (2015).

26) Kim H, Lee BH, Choi SH, Kim HJ, Jung SW, Hwang SH, Rhim H, Kim HC, Cho IH, Nah SY. Gintonin stimulates gliotransmitter release in cortical primary astrocytes. Neurosci. Lett., 603, 19-24 (2015).

27) Chapman KD, Dyer JM, Mullen RT. Biogenesis and functions of lipid droplets in plants: Thematic Review Series: Lipid Droplet Synthesis and Metabolism: from Yeast to Man. J. Lipid Res., 53, 215-226 (2012).

28) Horn PJ, Chapman KD. Lipidomics in tissues, cells and subcellular compartments. Plant J., 70, 69-80 (2012).

29) Goldshmit Y, Munro K, Leong SY, Pébay A, Turnley AM. LPA receptor expression in the central nervous system in health and following injury. Cell Tissue Res., 341, 23-32 (2010).

30) Tigyi G. Aiming drug discovery at lysophosphatidic acid targets. Br. J. Pharmacol., 161, 241-270 (2010).

31) Kim BJ, Nam JH, Kim KH, Joo M, Ha TS, Weon KY, Choi S, Jun JY, Park EJ, Wie J, So I, Nah SY. Characteristics of gintonin-mediated membrane depolarization of pacemaker activity in cultured interstitial cells of Cajal. Cell. Physiol. Biochem., 34, 873-890 (2014).

32) Millar AA, Smith MA, Kunst L. All fatty acids are not equal: discrimination in plant membrane lipids. Trends Plant Sci., 5, 95-101 (2000).

33) Pagès $C$, Simon MF, Valet $P$, Saulnier-Blache JS. Lysophosphatidic acid synthesis and release. Prostaglandins Other Lipid Mediat., 64, $1-10$ (2001). 